\title{
sensors
}

ISSN 1424-8220

(C) 2002 by MDPI

http://www.mdpi.net/sensors

\section{Surface Plasmon Resonance Studies on Molecular Imprinting}

\author{
Ping Li ${ }^{1, *}$, Yan Huang ${ }^{1}$, Junze $\mathrm{Hu}^{1}$, Chunwei Yuan ${ }^{1}$ and Baoping Lin ${ }^{2}$ \\ ${ }^{1}$ National Laboratory of Molecular \& Biomolecular Electronics, Southeast University, Nanjing \\ 210096, P. R. China. Tel: (86)-025-3794310, Fax: (86)-025-3619983. \\ ${ }^{2}$ Department of Chemistry and Chemical Engineering, Southeast University, Nanjing 210096, \\ P. R. China
}

* Author to whom correspondence should be addressed. E-mail: liping@seu.edu.cn

Received: 3 December 2001 / Accepted: 17 January 2002 / Published: 19 January 2002

\begin{abstract}
The molecular imprinted polymer (MIP) members were fabricated with the print molecule L-phenylalanine ethyl ester. The elution and adsorption procedures were investigated by surface plasmon resonance in situ. The changes of refractive angle during elution procedure suggest that the MIP is prepared on the base of the non-covalent interactions. This MIP member sensor can achieve enantioselective recognition.
\end{abstract}

Keywords: Molecular imprinting; Surface plasmon resonance; Elution; Adsorption

\section{Introduction}

Molecular imprinting is an easy and effective polymerization way to prepare synthetic materials able to mimic the molecular recognition in living system [1-3]. In this technique, the target molecule is associated with functional monomers to form a 'complex', which is then incorporated by polymerization into a highly cross-linked macroporous polymer matrix. Extraction of the print molecule leaves sites in the polymer with specific shape and functional group complementary to the original print molecule. Because of the special recognition capacity, the molecular imprinting polymer (MIP) has been widely investigated in the preparation of chiral chromatographic stationary phases, selective catalyst, and sensor components [4-7]. With surface plasmon resonance (SPR) optical method [8], the removal of the printed molecules from the polymer matrix and the rebinding of the template molecules to the polymer can be detected. We observed that the imprinted polymer members changed after the elution process. With three kinds of elution, the different SPR spectra were got. To the template molecular L-phenylalanine ethyl ester, the SPR spectra showed the adsorption capacity of 
MIP member to the printed molecules; to the template's enantiomer D-phenylalanine ethyl ester, the SPR spectra changed little.

\section{Experimental}

The crosslinker 1,4-butanediyl diacrylate (BDDA) and the photoinitator 2,4,6-trimethybenzol phenyphosphinate (Lucinrin LR 8893X) were obtained from BASF chemical (BASF, Germany). The functional monomer $\alpha$-methylacrylic acid (MAA), L- and D-phenylalanine were purchased from Shanghai Chemical (China). Other chemicals were analytical grade. The print molecule, Lphenylalanine ethyl ester (L-PHET) was synthesized as previously described [9].

The mixtures of template molecule L-PHET, functional monomer MAA, crosslinker BDDA and photoinitiator Lucinrin LR $8893 \mathrm{X}$ in $15 \mathrm{ml}$ methanol, were spread on the Au/glass substrates by spinning coating technique and then were exposed under UV irradiation for 4 hours with the light wavelength of $365 \mathrm{~nm}$. The temperature was controlled at $0{ }^{0} \mathrm{C}$. The deposited members were listed in Table1 with different contents of components. The polymer members were then eluted with various elutions: mole ratio of actontrile and acetic acid is (a) $9 / 1$, (b) $6 / 1$, (c) $3 / 1$ respectively. The uptake of enantiomers of PHET to the member B was detected: the mobile solutions were acetontrile-acetic $\operatorname{acid}\left(95 / 5\right.$, mole ration) with $0.5 \mathrm{~g} \mathrm{~L}^{-1} \mathrm{D}-\mathrm{PHET}$, or $0.5 \mathrm{~g} \mathrm{~L}^{-1}, 1.0 \mathrm{~g} \mathrm{~L}^{-1} \mathrm{~L}-\mathrm{PHET}$.

Table 1. Molecular Imprinted Polymer (MIP) Members (mmol).

\begin{tabular}{|c|c|c|c|c|}
\hline Member & L-PHET & MAA & BDDA & L.R. \\
\hline A & 0 & 4 & 20 & 0.3 \\
\hline B & 1 & 4 & 20 & 0.3 \\
\hline
\end{tabular}

SPR apparatus was constructed to experimentally investigate the optical properties of a chemical sample with surface plasmon waves. Any change of member refractive index and thickness can be detected in situ with high sensitivity.

\section{Results and Discussion}

Polymer member A, prepared without template molecule, was detected by SPR. The SPR spectrum was generated by changing the incident angle and recording the reflectivity. From the spectrum in Fig. 1, the thickness and refractive index of member A can be calculated as $75 \mathrm{~nm}$ and 1.42 by Freshel method. Elution (c) was injected into the small flow cell in the SPR. After washing for 3 hours with elution, it is found that the SPR spectrum remains unchanged, which indicates that the member thickness and structure keep stable during washing process, and the elution cannot influence the member.

Member B was prepared with L-PHET as the template molecule, and the SPR spectrum was shown as curve (a) in Fig.2. The thickness and the refractive index of member B can be calculated as $85 \mathrm{~nm}$ 
and 1.47. After washing with elution (c) for 6 hours, curve (b) was got in Fig.2. The resonance angle shifts to lower value. With Freshel methods, the thickness and refractive index of member after elution can be calculated as $85 \mathrm{~nm}$ and 1.42 . It is suggested that although the thickness of member B did not change, the member structure has been damaged by elution. During the polymerization process, the print molecule and the MAA monomer are supposed to interact by formation of pairs between the positively charged amino group of the print molecule and the negatively charged carboxyl group of the acrylic acid monomer. When MIP members washed by elution, the print molecules have stronger hydro bond interaction with acetic acid molecules in elution than with the functional monomer. The LPHET was washed away from MIP and leaves cavities in members, resulting in changes of resonance angle.

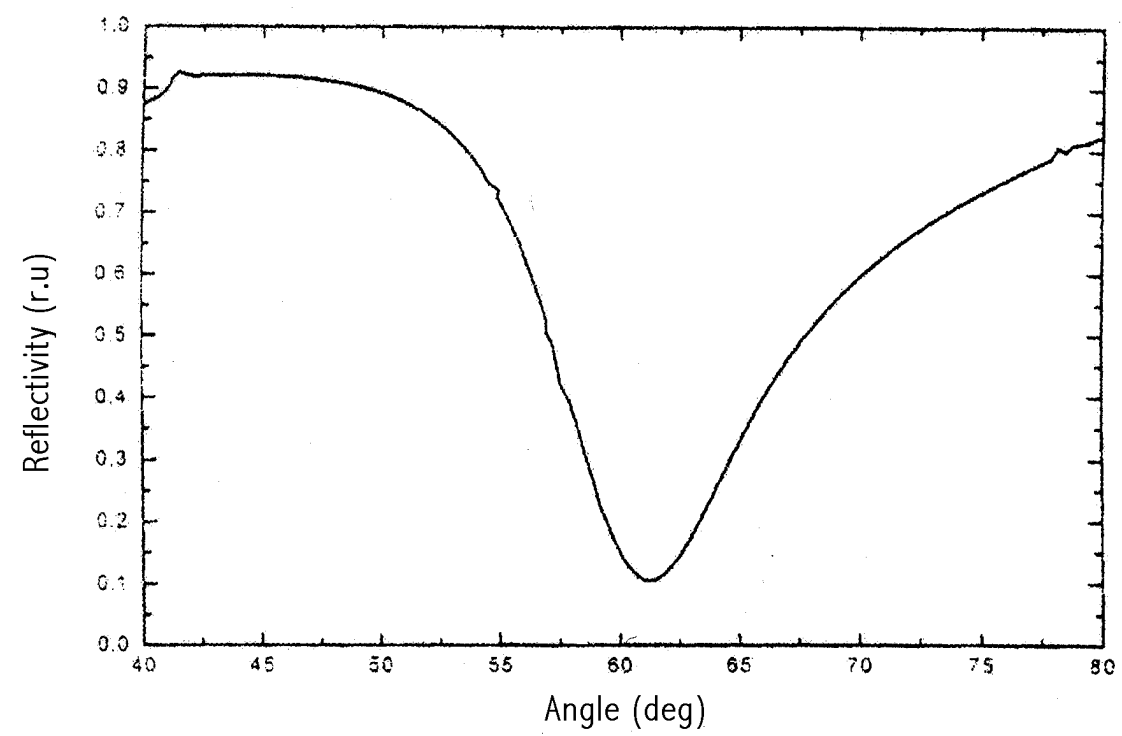

Figure 1. The SPR spectrum of member A remains unchanged after washing process.

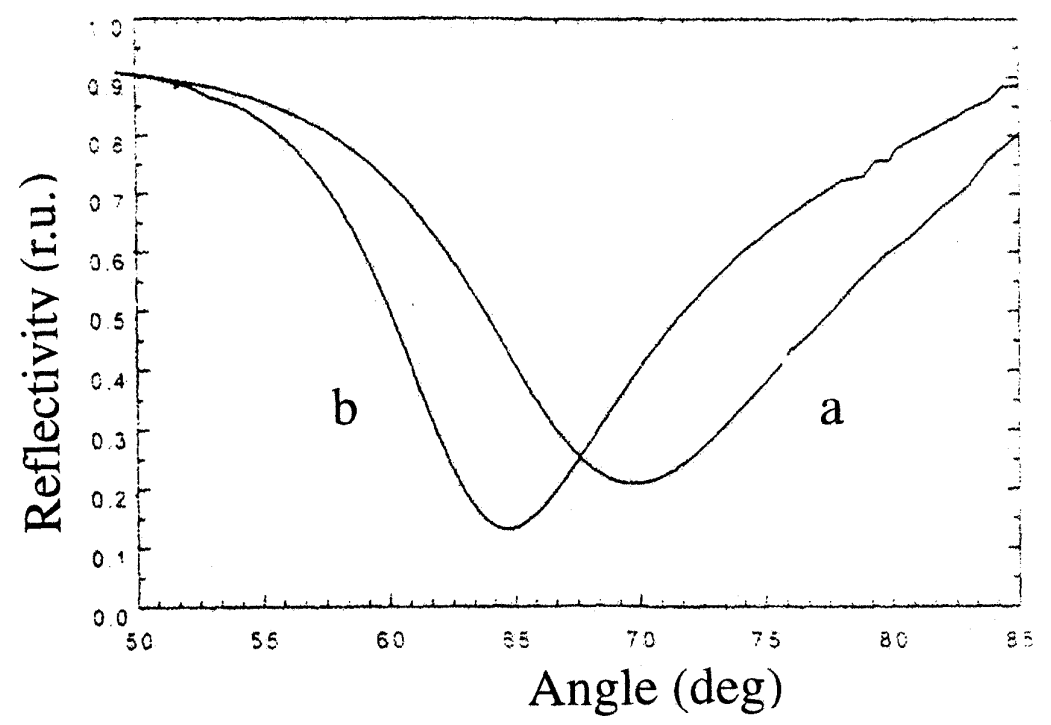

Figure 2. The SPR spectra of member B before (a) and after (b) washing process. 
Member B was washed with elutions (a), (b) and (c), which have different content of acetontrile. Fig. 3 shows changes of resonance angle of member B vs. the washing time under different elutions. It is found that the resonance angle changes dramatically with the increasing concentration of acetontrile in the mixture solution. The results are related with the polarity of solution. With higher polarity, the hydro bond interaction and ionic force are stronger between the elution and the L-PHET molecules, and the L-PHET can be easily taken away by elution. Elution (c) has the highest concentration of acetic acid, the tarnsferation balance of print molecules between the polymer and liquid phase is completed in shortest time. We can suggest that it is the non-covalent interactions that keep the print molecule L-PHET inside the MIP matrix.

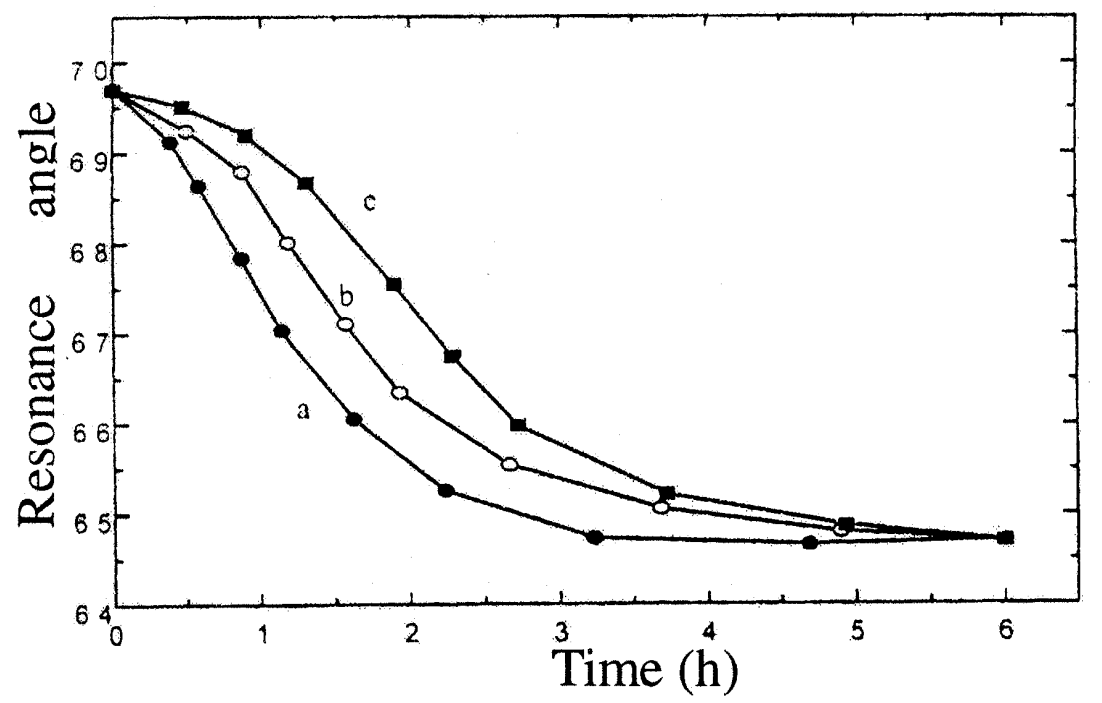

Figure 3. The changes of resonance angle of member with different elution: acetontrileactic acid (a) 9/1(mole ration), (b) 6/1, (c) 3/1.

We have observed the washing process in real time using SPR. From the spectra, we know that elution has no influence on the polymer member that prepared without imprinted molecule, but apparent influence on that prepared with imprinted molecule. The interactions between the print molecule and the functional monomer could be damaged by acetic acid, which means that the MIP is prepared on the base of the non-covalent interaction. And with SPR, we could get optimum elution for washing out the L-PHET. In our future work, we would use the SPR to investigate the re-adsorption process of the print molecules.

When the elution was acetontrile-acetic acid with $0.5 \mathrm{~g} \mathrm{~L}^{-1} \mathrm{D}-\mathrm{PHET}$, the difference between the SPR spectra of before and after adsorption was very small, showed in Fig.4. It reveals that few DPHET molecules adsorbed to the L-PHET imprinted member. When the elution was $0.5 \mathrm{~g} \mathrm{~L}^{-1} \mathrm{~L}-\mathrm{PHET}$, the SPR spectrum moved to high value, which showed the member has strong recognition to the LPHET. When the concentratin of L-PHET was $1.0 \mathrm{~g} \mathrm{~L}^{-1}$, the SPR spectrum moved to 74.6 degree. The adsorption of member to L-PHET increased. The different adsorption capacity of member B to LPHET and D-PHET suggested its enantiomeric analysis function. 


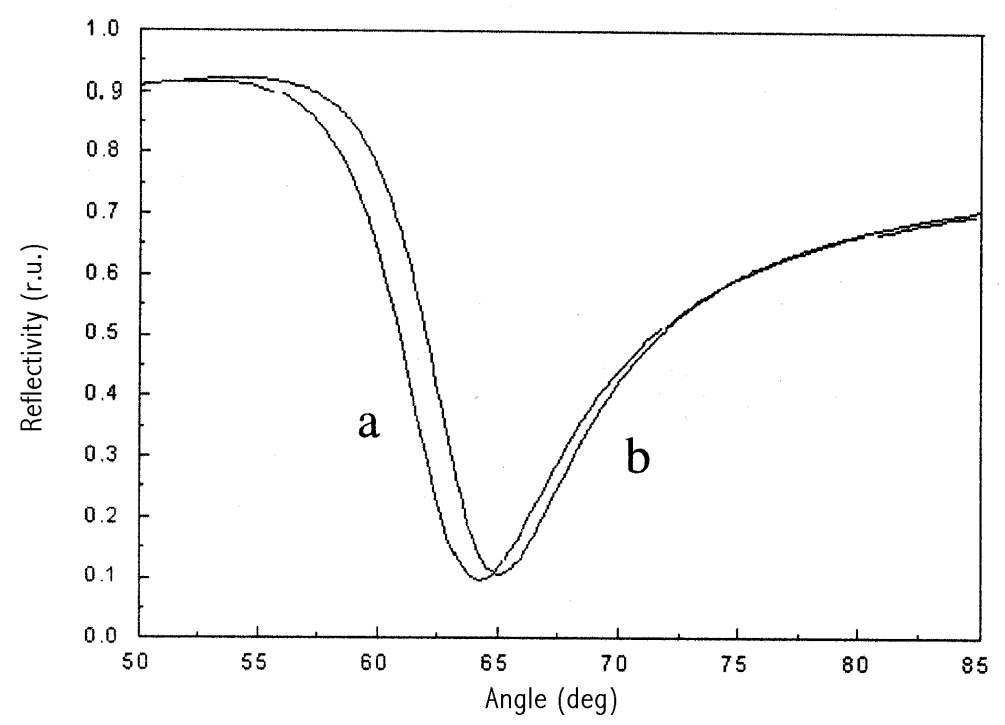

Figure 4. The SPR spectra of member B (a) after elution, (b) adsorption to $0.5 \mathrm{~g} \mathrm{~L}^{-1}$ D-PHET.

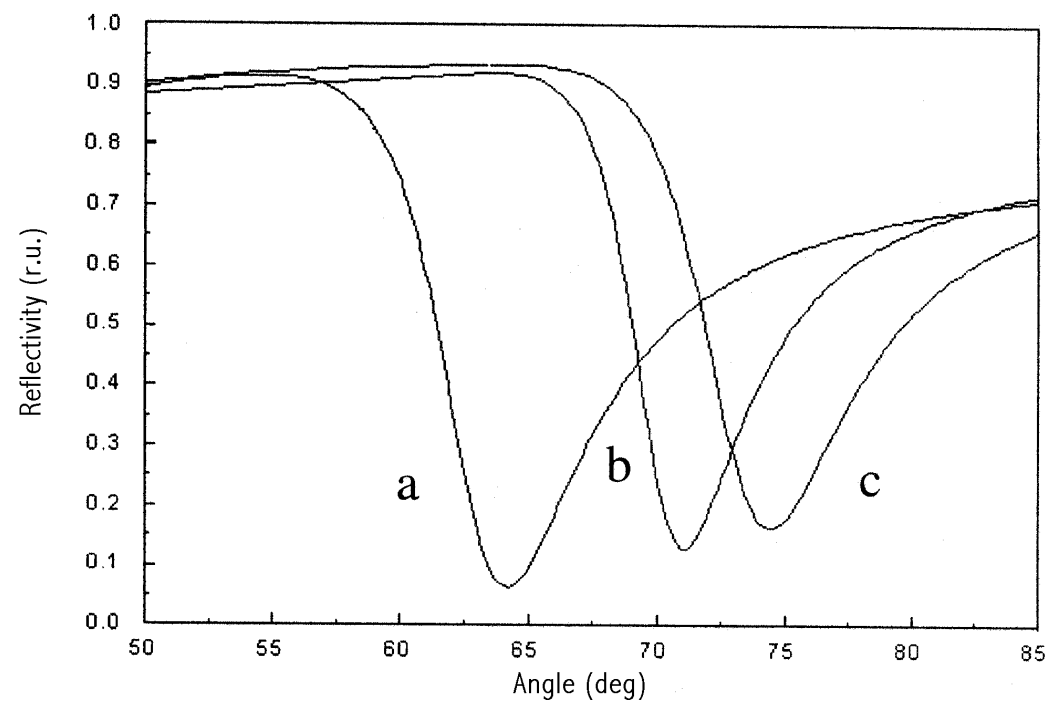

Figure 5. The SPR spectra of member B (a) after elution, (b) adsorption to $0.5 \mathrm{~g} \mathrm{~L}^{-1} \mathrm{~L}-\mathrm{PHET}$, (c) adsorption to $1.0 \mathrm{~g} \mathrm{~L}^{-1}$.

\section{Acknowledgements}

The work was supported by the Fundation for Ph.D Thesis Research of Chinese University (20010286006) and Natural Science Fundation of Jiangsu Province (BK2001015).

\section{References}

1. Wulff, G. Molecular Imprinting in Cross-Linked Materials with the Aid of Molecular Templates A Way towards Artificial Antibodies. Angew. Chem. Int. Ed. Engl. 1995, 34, 1812-1832. 
2. Haupt, K.; Mosbach, K. Molecularly Imprinted Polymers in Chemical and Biological Sensing. Biochem. Soc. Trans. 1999, 27 (2), 344-350.

3. Sellergren, B. Noncovalent Molecular Imprinting: Antibody-Like Molecular Recognition in Polymeric Network Materials. Trends Anal. Chem. 1997, 16(6), 310-320.

4. Hosoya, K.; Tanaka, N. Development of Uniform-Sized, Molecular-Imprinted Stationary Phases for HPLC. ACS Symp. Ser. 1998, 703, 143-158.

5. Takeuchi, T.; Haginaka, J. Separation and Sensing Based on Molecular Recognition Using Molecularly Imprinted Polymers. J. Chromatogr. B 1999, 728(1), 1-20.

6. Liu, Q.; Zhou, Y.X.; Liu, Y.T. Recent progresses in research on molecular imprinting sensors. Chin. J. Anal. Chem. 1999, 27(11), 1341-1347.

7. Dickert, F.L.; Lieberzeit, P.; Tortschanoff, M. Molecular imprints as artificial antibodies — a new generation of chemical sensors. Sensors and Actuators B: Chemical 2000, 65(1-3), 186-189.

8. Homola, J.; Sinclair, S. Yee Surface plasmon resonance sensors: review. Sensors and Acutors $B$ 1999, (54), 3-15.

9. Andersson, L.; Sellergren, B.; Mosbach, K. Imprinting of Amino Acid Derivatives in Macroporous Polymers. Tetrahed. Lett. 1984, 25(45), 5211-5214.

Sample Availability: Available from the authors.

(C) 2002 by MDPI (http://www.mdpi.net). Reproduction is permitted for noncommercial purposes. 